\title{
Elderly patients with glioblastoma: the impact of surgical resection extent on survival
}

\author{
Participants: \\ Marcelo Lemos Vieira da Cunha' \\ Augusto Cesar Santos Esmeraldo ${ }^{1}$ \\ Leonardo Augusto Wendling Henriques ${ }^{1}$ \\ Marcos Antônio Martins dos Santos Jr. ${ }^{1}$ \\ Roger Thomaz Rotta Medeiros ${ }^{1}$ \\ (iD) Ricardo Vieira Botelho ${ }^{\text {* }}$ \\ Final version: April 2018 \\ 1. Sociedade Brasileira de Neurocirurgia \\ *corresponding autor: bitbot@uol.com.br \\ http://dx.doi.org/10.1590/1806-9282.65.7.937
}

The Guidelines Project, an initiative of the Brazilian Medical Association, aims to combine information from the medical field in order to standardize producers to assist the reasoning and decision-making of doctors.

The information provided through this project must be assessed and criticized by the physician responsible for the conduct that will be adopted, depending on the conditions and the clinical status of each patient.

The impact of the neurosurgery in elderly patients with glioblastoma multiforme (GBM) is still unclear. The objective of this systematic review was to evaluate the overall survival of patients over 65 years old submitted to GBM surgical treatment and analyze the rates of postoperative complications in this population. A search on the Medline, Cochrane e Google Scholar electronic databases between January 2005 and April 2018 identified seven studies that evaluated the neurosurgical treatment of patients older than 65 years with GBM. Surgical procedures included complete or partial resection or tumor biopsy. In elderly GBM patients, total surgical resection of the tumor was associated with longer overall postoperative survival when compared with the partial resection or biopsy. Based on this study, neurosurgery is recommended to increase the overall survival of elderly patients with GBM and a good overall preoperative condition. The high rate of complications in this population should be taken into consideration for the surgical decision.

The details of the methodology of this guideline are set out in Annex I.

\section{INTRODUCTION}

Glioblastoma multiforme (GBM), the most common type of primary brain tumor in adults, is associated with a deeply unfavorable prognosis, with an estimated 5-year survival rate of only $10 \%$. Several prognostic factors associated with GBM have been evaluated in the medical literature, among them the patient's age and clinical performance consistently appear as having a negative influence on survival'. The estimated average survival of GBM patients older than 75 years is 2.5 months ${ }^{2}$.

More than one-third of patients newly diagnosed with GBM have are over 65 years old. As the population ages, neurosurgeons face an increasing probability of having to manage patients with GBM in increasingly advanced ages. However, while studies conducted in populations younger than 65 years favor the degree of resection as one of the main factors of better prognosis ${ }^{3.4}$, elderly patients are often excluded from clinical trials ${ }^{5}$; therefore, data assessing 
the importance of the neurosurgical resection degree in this age group are still illusory.

Based on these considerations, the aim of this study was to evaluate the effect of surgical treatment in elderly patients with GBM.

\section{RESULTS}

\section{Data Collection and Analysis}

In the study by Hoffermann et al., the morbidity associated with neurosurgery was $19.3 \%$. Permanent neurological deficits (defined by the authors as focal neurological deficits that interfere significantly in daily living activities) affected $12.1 \%$ of the cases in the study, while wound infections and healing problems affected $4 \%$ of them. As a whole, $3.2 \%$ of elderly patients with GBM treated with neurosurgery presented transitional medical complications (deep vein thrombosis, pulmonary embolism, pneumonia, or myocardial infarction $)^{6}(\mathrm{~B})$.

In the study by Oszvald et al., the disease-free survival in elderly patients was of 7.5 months. There was no statistical difference between total and partial resection. However, in the comparison of surgery (with partial or total resection) versus biopsy, there was a significative difference $(p<0.0027)(B)$.

The performance status of ECOG PS (Eastern Cooperative Oncology Group) (ECOG PS) in the series by Lombardi et al. was between $0-1$ in $82 \%$ of the elderly patients who underwent neurosurgery, while Harris et al.reported a smaller percentage (64.7\%) for the same ECOG PS group ${ }^{8}(\mathrm{~B})$.

In the study by Ewelt et al., the authors speculated that the clinical condition at the presentation was more important to the overall survival of patients of that age (Karnofsky performance score [KPS] $>$ 70, p $<0.001$; age $<75$ years, $p=0.224$ ). For the analysis, our study considered only data consistent with the neurosurgical therapy alone. But in the three comparison groups of the study (Group A = surgery alone; Group B = surgery + chemotherapy and Group C = surgery + radiotherapy + chemotherapy), the longest survival was with complete surgery (resection of the tumor uptake area) $)^{2}(\mathrm{~B})$.

Gerstein et al.: The authors found that the KPS at the time of surgery in elderly patients with GBM, as well as the degree of surgical resection were the most important factor related to survival time ${ }^{9}$ (B).

Pretanvil et al.: The authors reviewed the therapy used in elderly patients with GBM. This publica- tion included at least eight modalities of treatment, including types of neurosurgical resections (partial and total biopsy) and associations with chemotherapy and radiotherapy, evidencing the heterogeneity of therapy in elderly patients. When compared withyounger patients, the biopsy was more prevalent (38.3\%) among those aged $>70$ years (versus $24.9 \%$ in patients aged between 18-70 years). This treatment difference led to significantly lower survival in the group of elderly patients, considering the degree ofresection $^{10}(\mathrm{~B})$.

\section{Analysis of the combined results}

The grouped analysis of the seven studies that assessed total and partial surgical resection included 473 and 513 patients, respectively, while the four studies that evaluated biopsy as a neurosurgical treatment included 90 patients.

Overall, the mean age of patients was $71.98 \pm$ 4.40 years. The definition of "elderly patient" varied between the studies, but all reported results for patients over 60 years old. The mean survival (in months) of the group that underwent total resection was higher than that of the group that underwent partial resection, which, in turn, was greater than that of the group who underwent the biopsy (Figures 1, 2, and 3).

\section{Results according to tumor resection extent}

The mean combined overall survival time (COS) was 13.13 months (95\% CI 12.02-14.23 months) in patients in whom total resection was possible (Figure1), 7.52 months (95\% CI 6.94-8.11 months) in those who underwent partial resection (Figure 2), and 2.56 months (95\% CI 2.02-3.06 months) in patients who underwent biopsy alone (Figure 3).

The combined overall calculated survival heterogeneity (I2) of the for total resection, partial resection, and biopsy was $11.8,84.8$, and 78.8 , respectively. Forest Plot analysis (Figures 1, 2, and 3) showed that the results for the complete resection were more homogeneous than the other two possible resections.

\section{Complications}

Since surgery is not a definitive treatment for GBM, the complications associated with the therapy were often described for the treatment as a whole or as toxicity associated with radiotherapy and chemotherapy combined.

Ewelt et al. observed that almost $25 \%$ of the pa- 
FIGURE 1. FOREST PLOT OF THE OVERALL SURVIVAL RATES FOR TOTAL RESECTION.

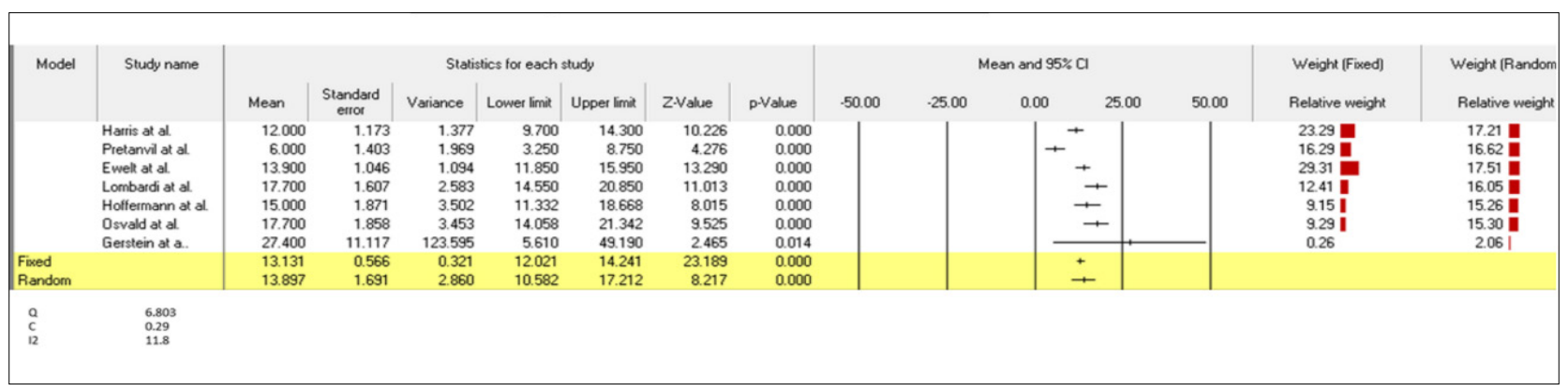

FIGURE 2. FOREST PLOT OF THE OVERALL SURVIVAL RATES FOR PARTIAL RESECTION.

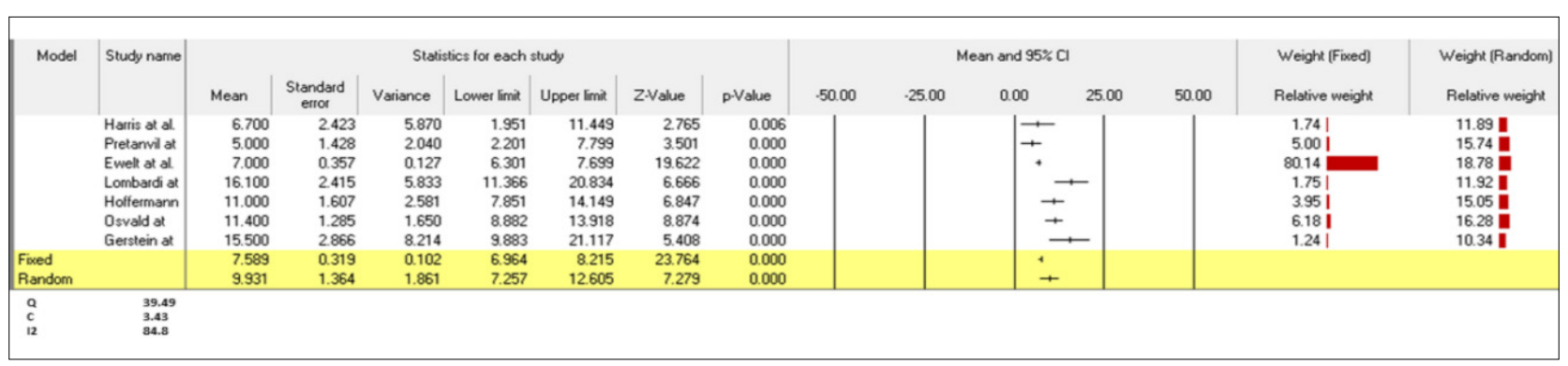

FIGURE 3. FOREST PLOT OF THE OVERALL SURVIVAL RATES FOR BIOPSY.

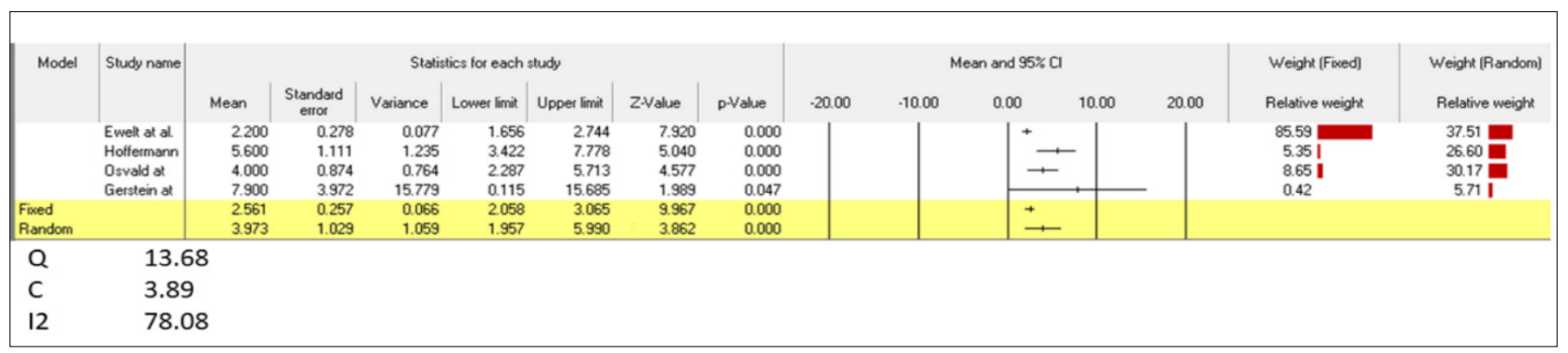

tients had complications after surgery: 21 infections, ten leakages of cerebrospinal fluid (CSF), and seven complications associated with CFS circulation defects. The authors did not consider the adverse events to have significantly affected the survival or the postoperative KPS in the population as a whole, although these observations did show a tendency toward significance $^{2}(B)$. The same result was demonstrated in a single-center study by Bohman et al. including 382 patients; the authors found no significant differences in survival in patients with GBM and postoperative infections ${ }^{11}(\mathrm{~B})$.

Harris et al. described the results of 108 patients; 35 were treated with the best supportive care, one received temozolomide (TMZ) alone, 40 received only radiotherapy, and 32 received radiotherapy and TMZ combined. Regarding the patients who received active treatment, 29 were hospitalized during the radio- therapy (38\%), 22 were hospitalized due to declining functional status related to the disease and seven were hospitalized due to other active medical problems (including infection and comorbidities). None of the patients was admitted to the hospital due to toxicities related to the treatment ${ }^{12}(\mathrm{~B})$.

Lombardi et al. describe only toxicities associated with treatment. In relation to non-hematological toxicities, the authors observed six patients $(2 \%)$ with grade 3-4 toxicity (two with asthenia, two with increased transaminases, one with nausea, and one with intestinal perforation). A total of 53 patients (22\%) received reduced or delayed TMZ during the treatment due to toxicities related to the treatment; among them, $28 \%$ and $20 \%$ were patients who received radiotherapy with 40 Gy and 60 Gy, respectively, but no fatal toxicity associated with the therapy was observed $^{8}(\mathrm{~B})$. The authors did not consider the neurolo- 
gical side effects and the dexamethasone dose during radiotherapy and observed grade 3-4 risk factors in $7 \%$ of all patients.

Hofferman et al. briefly described their resultsin relation to complications, pointing that the surgical mortality was $4.0 \%$ and the global morbidity was $19.3 \%{ }^{6}(\mathrm{~B})$.

Oszvald et al. ${ }^{7}(\mathrm{~B})$ did not describe complications, while Gerstein et al. ${ }^{9}(\mathrm{~B})$ described toxicities related to therapy. Radiotherapy could be completed in just $59 \%$ of the patients. Hematological toxicity was found in $7 \%$ of the patients and non-hematologic toxicities in $14 \%$. During radiotherapy, one patient had pneumonia, and four others had a progression of the disease and were not able to continue the treatment. Other nine patients presented cytopenia, two had pneumonia, three had rashes, and two showed high levels of transaminases. These were not surgical complications, but complications presented during the global treatment for GBM.

\section{DISCUSSION}

In general, the population is aging and the number of elderly patients with GBM is increasing. Studies show that up to $40 \%$ of patients with GBM are over 65 years old ${ }^{7,8,10}$.

For patients over 65 years old and with high-grade gliomas, the negative outcomes associated with surgery have been regarded as a direct consequence of advanced age. The hypotheses proposed to explain these negative results include increased perioperative and postoperative comorbidities, due to age and comorbidities, and reduced tolerance to the therapeutic procedures. In addition, neurodegeneration, resistance to radiotherapy and chemotherapy, different histological types, and genetic mutations are also possible factors that could explain the reduced survival in this population. In several trials on cancer therapy, age is one of the exclusion criteria.

A question that remains is whether age, as an isolated factor, could have such a strong influence on the surgical outcome. The plethora of different treatments offered to patients with GBM makes it difficult to have an a priori analysis of the effect of the treatment in elderly patients. In addition, experience suggests that elderly patients with GBM are less tolerant of parallel treatments in comparison with younger patients. However, some authors sug- gest that elderly patients in good clinical conditions should receive treatment similar to that of younger patients ${ }^{10,12,13}$.

The existing need to clarify the effect of surgical treatment in elderly patients with GBM was the reason for this analysis.

This review found seven studies that included 1,076 elderly patients with GBM. Of these, 473 were submitted to total resection, 513 underwent partial resection, and 90 were submitted to biopsy. Logically, the possibility and extent of the resection do not depend exclusively on the surgical technique, but also on the degree of tumor involvement and invasion of the cerebral parenchyma. Thus, the presentation of patients is essential to define their prognosis.

Our review suggests that the total resection of the tumor is beneficial to the overall postoperative survival of the patient when compared to the partial resection or biopsy. The life expectancy of patients who underwent partial resection and biopsy was relatively short (7.52 months and 2.56 months, respectively).

Other potentially desirable results for analysis in this review could not be evaluated since they often were not been described in the primary studies. It is worth noting that it would be interesting to assess the neurological status before and after the interventions. These could be related to survival and, very likely, to the quality of life of the patients treated. In patients with severe impairment of cognitive functions, life expectancy is not the best element to be assessed. Even so, these data were not reported in many studies, which makes it difficult to interpret them.

Another limitation of this study is the fact that patients undergoing neurosurgical therapy are subjected to different treatment plans with various regimens of chemotherapy and radiotherapy during the follow-up, making it impossible to compare the therapies.

The complication rates of the studies assessed ranged from $19 \%$ to $25 \%^{2.6}$. These data should be taken into consideration when surgery is recommended for elderly patients with GBM.

Other models for global analysis that compare the risk rates and the survival curves per treatment regimen have been used as a model for combined analyzes. All studies identified were non-comparative, missing, therefore, comparisons between the 
treatments. In addition to these limitations, the wide variation of chemotherapy and radiotherapy regimens associated to surgery that have been described in the literature may have a potential bias in the evaluation of the effect of surgery GBM treatment in elderly patients.

Practical aspects of management perhaps are more important in elderly patients as pre-morbid capabilities, such as mobility, communication, memory, and other intellectual functions. Tumor resection would be beneficial for survival if these functions could be improved with the resection of the tumor. However, these data are not described in the literature.

A total and safe surgical resection is suggested as the ideal treatment for the GBM. The value of this recommendation for the elderly population was not clear until the present moment. Now, our study has shown a benefit in overall survival (OS) associated with the total resection of the tumor in older individuals, even though the rate of postoperative complications was substantial in some series.

\section{Research implications}

New prospective studies should be performed to confirm the results found in this review. Specific evaluations in eloquent areas and deep tumors should be performed.

\section{Practical implications}

Similarly to the current recommendation for adults younger than 60-year-old with GBM, the total resection of the tumor should be the therapeutic goal in patients over 60 years olwwd in whom the resection is feasible, provided the clinical conditions allow.

\section{RECOMMENDATION}

Considering that (1) all the studies included in this review were case series (very low quality evidence), (2) GBM is a fatal disease with a poor prognosis, (3) the total macroscopic removal of the tumor has been associated with greater longevity than its partial removal or biopsy, (4) no alternative treatment other than surgery is available at the moment, and (5) the morbidity associated with surgery is relatively high, surgery is recommended to increase the overall survival in elderly patients with GBM and a condition compatible with low pre-operative morbidity (low to moderate strength of recommendation).

\section{ANNEX I}

\section{Clinical question}

What is the impact of the surgical resection extent in the survival of elderly patients with glioblastoma?

\section{Eligibility criteria}

- Types of study: According to the hierarchy of evidence, our initial goal was to include randomized studies and, in their absence, comparative controlled studies or cohort studies describing the outcome of surgery in the target population (elderly patients with GBM).

- Types of participants/patients: Elderly individuals aged over 65 years. The review included only studies with that treated new GBM, while studies on recurrent GBM were excluded.

- Types of intervention: Our objective was to evaluate the (neuro)surgical treatment outcome. The results were described and compared according to the degree of resection, as total or partial resection or biopsy.

- Types of outcome measures: We evaluated the clinical outcome, neurological status, quality of life, survival, and disease-free survival.

The evaluation also included the complications reported.

\section{Search for papers}

\section{Database}

Electronic searches were conducted in the Medline, Cochrane Controlled Trials Register,, and Google Scholar databases using the following key terms:

("glioblastoma"[MeSH Terms] OR "glioblastoma"[All Fields] OR ("glioblastoma"[All Fields] AND "multiforme"[All Fields]) OR ("glioblastoma multiforme"[All Fields]) AND (older[All Fields]) OR ("aged"[MeSH Terms] OR "aged"[All Fields] OR "elderly"[All Fields]) AND ("surgery"[Subheading] OR "surgery"[All Fields] OR "surgical procedures, operative"[MeSH Terms] OR ("surgical”[All Fields] AND "procedures"[All Fields] AND “operative"[All Fields]) OR "operative surgical procedures"[All Fields] OR "surgery"[All Fields] OR "general surgery"[MeSH Terms] OR ("general"[All Fields] AND "surgery"[All Fields]) OR "general surgery"[All Fields]) OR ("surgical procedures, operative"[MeSH Terms] OR ("surgical"[All Fields] AND "procedures"[All Fields] AND "operative"[All Fields]) OR "operative surgical procedures"[All Fields] OR "surgical”[All Fields]) OR resection [All Fields]) AND “humans”[MeSH Terms]. 


\section{Identification of descriptors}

\begin{tabular}{|l|}
\hline $\mathbf{P}$ - glioblastoma \\
\hline $\mathbf{I}$ - surgery, surgical procedure \\
\hline $\mathbf{C}$ - not determined \\
\hline $\mathbf{O}$ - impact, quality of life, survival, neurological status \\
\hline
\end{tabular}

\section{Data collection}

We determined the year 2005 as the initial year of publication of articles for this study since this was the year when the Protocol Stupp, which is currently used as a guide for high-grade glioma therapy, was established. We identified a total of 1,960 titles between January 2005 and April 2018.

The search strategy was outlined by five authors, and the selection of the articles was performed by two independent authors. Disagreements were resolved by discussion between the authors.

\section{Critical evaluation}

Relevance - clinical importance

This guideline was prepared by means of a clinically relevant question in order to gather information in medicine to standardize approaches and assist in decision-making.

\section{Methodological Analysis}

The methodological quality of the studies included in the analysis was assessed using a validated and revised version of the Minors tool (Methodological Index for Non-Randomized Studies). The items assessed were the following:

1. A clear of the study, established a priori. The objective of the research should be relevant and accurate in light of the literature available.

2. Consecutive inclusion of patients in the study.
All patients who met the inclusion criteria should be included in the study during the period of evaluation (without exclusions, or the reasons for exclusions of patients recruited must be detailed in the article).

3. Prospective data collection: implies that data must be collected in accordance with a protocol established before the beginning of the study.

4. Appropriate endpoints to the objective of the study. Clear explanation (without ambiguity) of the criteria used to assess the main outcome, in accordance with the objective of the study. The results should be assessed on an intention-to-treat analysis.

5. Unbiased assessment of the study endpoints: Independent assessment (blind) of the endpoints or double-blind assessment of subjective results. The reasons for unblinded assessments must be declared.

6. Appropriate follow-up period to the objectives of the study. The studies should have a sufficient period of follow-up for the proper evaluation of the outcomes and adverse events.

7. Loss of follow-up not exceeding $5 \%$ of the sample treated. All patients must be included in the assessment.

8. Prospective calculation of sample size included in the study. Difference information to detect and calculate the 95\% confidence intervals(ICs), in accordance with the expected incidence of endpoints and information on the level of statistical significance and power estimation in the comparison of outcomes.

The evaluation of the methodological quality of the studies is described in Table 1 . The quality of the evidence was evaluated according to the Grading of Recommendations, Assessment, Development, and Evaluations (Grade) ${ }^{14}$.

TABLE 1. EVALUATION OF THE METHODOLOGICAL QUALITY OF THE STUDIES INCLUDED IN THE ANALYSIS.

\begin{tabular}{|c|c|c|c|c|c|c|c|c|c|}
\hline $\begin{array}{l}\text { AUTHORS \& } \\
\text { YEAR }\end{array}$ & $\begin{array}{l}\text { Clearly } \\
\text { stated } \\
\text { aim }\end{array}$ & $\begin{array}{l}\text { Inclusion of } \\
\text { consecutive } \\
\text { patients }\end{array}$ & $\begin{array}{l}\text { Prospective } \\
\text { data collec- } \\
\text { tion }\end{array}$ & $\begin{array}{l}\text { Endpoints } \\
\text { appropriate } \\
\text { to study } \\
\text { aim }\end{array}$ & $\begin{array}{l}\text { Unbiased } \\
\text { assessment } \\
\text { of study } \\
\text { endpoint }\end{array}$ & $\begin{array}{l}\text { Follow-up } \\
\text { period ap- } \\
\text { propriate to } \\
\text { study aim }\end{array}$ & $\begin{array}{l}<5 \% \text { lost to } \\
\text { follow-up }\end{array}$ & $\begin{array}{l}\text { Prospective } \\
\text { calculation } \\
\text { of study } \\
\text { size }\end{array}$ & Total \\
\hline HARRIS (2017) & 2 & 2 & 2 & 2 & 2 & 2 & 2 & 1 & 15 \\
\hline $\begin{array}{l}\text { PRETANVIL } \\
(2017)\end{array}$ & 2 & 2 & 2 & 2 & 2 & 2 & 2 & 1 & 15 \\
\hline EWELT (2011) & 2 & 2 & 2 & 2 & 2 & 2 & 2 & 1 & 15 \\
\hline $\begin{array}{l}\text { LOMBARDI } \\
(2015)\end{array}$ & 2 & 2 & 2 & 2 & 2 & 2 & 2 & 1 & 15 \\
\hline $\begin{array}{l}\text { HOFFERMANN } \\
(2015)\end{array}$ & 2 & 2 & 2 & 2 & 2 & 2 & 2 & 1 & 15 \\
\hline OSZVALD (2012) & 2 & 2 & 2 & 2 & 2 & 2 & 2 & 1 & 15 \\
\hline GERSTEIN (2010) & 2 & 2 & 2 & 2 & 2 & 2 & 2 & 1 & 15 \\
\hline
\end{tabular}




\section{Statistical analysis}

Data are presented as descriptive statistics using summary measures (mean, standard deviation, median, minimum, and maximum). The standard deviations were calculated using the confidence intervals (CI). All tests were performed with a significance level of 5\%. The analyses were carried out using a 2006 version of the Comprehensive Meta-Analysis (CMA) software (Statplus, Englewood, NJ, USA).

\section{RESULTS}

From the titles retrieved in the search, 75 articles were identified that addressed the treatment of GBM specifically in elderly patients. After an individual analysis of 75 Abstracts, 26 articles were selected on the neurosurgical treatment of elderly patients with GBM. In seven of these studies, we obtained results of the surgical treatment of GBM in elderly patients with total and partial resection and only four studies with biopsy, all of them with a retrospective cohort design.

The data were tabulated and analyzed (Table 2).

The results obtained from the search strategy are described in the Prisma flowchart (Figure 4).

The seven studies included in the analysis had a retrospective design and included elderly patients who underwent surgery due to GBM. The objective of each

TABLE 2. PREFERRED REPORT ITEMS FOR SYSTEMATIC REVIEW AND META-ANALYSIS CHECKLIST (PRISMA).

\begin{tabular}{|c|c|c|c|}
\hline Section/Topic & \# & Checklist item & $\begin{array}{l}\text { Reported on } \\
\text { page \# }\end{array}$ \\
\hline \multicolumn{4}{|l|}{ TITLE } \\
\hline Title & 1 & Identify the report as a systematic review, meta-analysis, or both. & 1 \\
\hline \multicolumn{4}{|l|}{ ABSTRACT } \\
\hline Structured summary & 2 & $\begin{array}{l}\text { Provide a structured summary including, as applicable: background; objectives; } \\
\text { data sources; study eligibility criteria, participants, and interventions; study } \\
\text { appraisal and synthesis methods; results; limitations; conclusions and implications } \\
\text { of key findings; systematic review registration number. }\end{array}$ & $2-3$ \\
\hline \multicolumn{4}{|l|}{ INTRODUCTION } \\
\hline Rationale & 3 & Describe the rationale for the review in the context of what is already known & 4 \\
\hline Objectives & 4 & $\begin{array}{l}\text { Provide an explicit statement of questions being addressed with reference to } \\
\text { participants, interventions, comparisons, outcomes, and study design (PICOS). }\end{array}$ & $4-8$ \\
\hline \multicolumn{4}{|l|}{ METHODS } \\
\hline $\begin{array}{l}\text { Protocol and } \\
\text { registration }\end{array}$ & 5 & $\begin{array}{l}\text { Indicate if a review protocol exists, if and where it can be accessed (e.g., Web address), } \\
\text { and, if available, provide registration information including registration number. }\end{array}$ & $\begin{array}{l}\text { Not applicable } \\
\text { to this study }\end{array}$ \\
\hline Eligibility criteria & 6 & $\begin{array}{l}\text { Specify study characteristics (e.g., PICOS, length of follow_up) and report } \\
\text { characteristics (e.g., years considered, language, publication status) used as criteria } \\
\text { for eligibility, giving a rationale. }\end{array}$ & 4-8 and Table 1 \\
\hline Information sources & 7 & $\begin{array}{l}\text { Describe all information sources (e.g., databases with dates of coverage, contact } \\
\text { with study authors to identify additional studies) in the search and date last } \\
\text { searched }\end{array}$ & 4-8 and Table 1 \\
\hline Search & 8 & $\begin{array}{l}\text { Present full electronic search strategy for at least one database, including any limits } \\
\text { used, such that it could be repeated. }\end{array}$ & 5 \\
\hline Study selection & 9 & $\begin{array}{l}\text { State the process for selecting studies (i.e., screening, eligibility, included in the } \\
\text { systematic review, and, if applicable, included in the meta_analysis). }\end{array}$ & $4-5$ \\
\hline $\begin{array}{l}\text { Data collection } \\
\text { process }\end{array}$ & 10 & $\begin{array}{l}\text { Describe the method of data extraction from reports (e.g., piloted forms, } \\
\text { independently, in duplicate) and any processes for obtaining and confirming data } \\
\text { from investigators }\end{array}$ & $4-5$ \\
\hline Data items & 11 & $\begin{array}{l}\text { List and define all variables for which data were sought (e.g., PICOS, funding } \\
\text { sources) and any assumptions and simplifications made. }\end{array}$ & $4-5$ \\
\hline $\begin{array}{l}\text { Risk of bias in } \\
\text { individual studies }\end{array}$ & 12 & $\begin{array}{l}\text { Describe methods used for assessing the risk of bias of individual studies } \\
\text { (including specification of whether this was done at the study or outcome level), } \\
\text { and how this information is to be used in any data synthesis. }\end{array}$ & $\begin{array}{l}\text { Not applicable } \\
\text { to this study }\end{array}$ \\
\hline Summary measures & 13 & State the principal summary measures (e.g., risk ratio, difference in means). & 8 \\
\hline Synthesis of results & 14 & $\begin{array}{l}\text { Describe the methods of handling data and combining results of studies, if done, } \\
\text { including measures of consistency (e.g., 12) for each meta_analysis. }\end{array}$ & $\begin{array}{l}\text { Not applicable } \\
\text { to this study }\end{array}$ \\
\hline $\begin{array}{l}\text { Risk of bias across } \\
\text { studies }\end{array}$ & 15 & $\begin{array}{l}\text { Specify any assessment of the risk of bias that may affect the cumulative evidence } \\
\text { (e.g., publication bias, selective reporting within studies }\end{array}$ & $\begin{array}{l}\text { Not applicable } \\
\text { to this study }\end{array}$ \\
\hline Additional analyses & 16 & $\begin{array}{l}\text { Describe methods of additional analyses (e.g., sensitivity or subgroup analyses, } \\
\text { meta-regression), if done, indicating which were pre_specified }\end{array}$ & $\begin{array}{l}\text { Not applicable } \\
\text { to this study }\end{array}$ \\
\hline
\end{tabular}




\begin{tabular}{|c|c|c|c|}
\hline Section/Topic & \# & Checklist item & $\begin{array}{l}\text { Reported on } \\
\text { page \# }\end{array}$ \\
\hline \multicolumn{4}{|l|}{ RESULTS } \\
\hline Study selection & 17 & $\begin{array}{l}\text { Give numbers of studies screened, assessed for eligibility, and included in the } \\
\text { review, with reasons for exclusions at each stage, ideally with a flow diagram }\end{array}$ & $\begin{array}{l}6 \text { and flow } \\
\text { diagram }\end{array}$ \\
\hline Study characteristics & 18 & $\begin{array}{l}\text { For each study, present characteristics for which data were extracted (e.g., study } \\
\text { size, PICOS, follow-up period) and provide the citations. }\end{array}$ & Table 1 \\
\hline $\begin{array}{l}\text { Risk of bias within } \\
\text { studies }\end{array}$ & 19 & $\begin{array}{l}\text { Present data on the risk of bias of each study and, if available, any outcome level } \\
\text { assessment (see item 12). }\end{array}$ & $\begin{array}{l}\text { Not applicable } \\
\text { to this study }\end{array}$ \\
\hline $\begin{array}{l}\text { Results of individual } \\
\text { studies }\end{array}$ & 20 & $\begin{array}{l}\text { For all outcomes considered (benefits or harms), present, for each study: (a) simple } \\
\text { summary data for each intervention group (b) effect estimates and confidence } \\
\text { intervals, ideally with a forest plot }\end{array}$ & $\begin{array}{l}\text { Not applicable } \\
\text { to this study }\end{array}$ \\
\hline Synthesis of results & 21 & $\begin{array}{l}\text { Present results of each meta-analysis done, including confidence intervals and } \\
\text { measures of consistency. }\end{array}$ & $\begin{array}{l}\text { Not applicable } \\
\text { to this study }\end{array}$ \\
\hline $\begin{array}{l}\text { Risk of bias across } \\
\text { studies }\end{array}$ & 22 & Present results of any assessment of the risk of bias across studies (see Item 15). & $\begin{array}{l}\text { Not applicable } \\
\text { to this study }\end{array}$ \\
\hline Additional analysis & 23 & $\begin{array}{l}\text { Give results of additional analyses, if done (e.g., sensitivity or subgroup analyses, } \\
\text { meta-regression [see Item 16]). }\end{array}$ & $\begin{array}{l}\text { Not applicable } \\
\text { to this study }\end{array}$ \\
\hline \multicolumn{4}{|l|}{ DISCUSSION } \\
\hline Summary of evidence & 24 & $\begin{array}{l}\text { Summarize the main findings including the strength of evidence for each main } \\
\text { outcome; consider their relevance to key groups (e.g., healthcare providers, users, } \\
\text { and policy makers). }\end{array}$ & $11-14$ \\
\hline Limitations & 25 & $\begin{array}{l}\text { Discuss limitations at study and outcome level (e.g., risk of bias), and at review- } \\
\text { level (e.g., incomplete retrieval of identified research, reporting bias). }\end{array}$ & 12 \\
\hline Conclusions & 26 & $\begin{array}{l}\text { Provide a general interpretation of the results in the context of other evidence, and } \\
\text { implications for future research. }\end{array}$ & $12-14$ \\
\hline \multicolumn{4}{|l|}{ FUNDING } \\
\hline Funding & 27 & $\begin{array}{l}\text { Describe sources of funding for the systematic review and other support (e.g., } \\
\text { supply of data); the role of funders for the systematic review. }\end{array}$ & 14 \\
\hline
\end{tabular}

FIGURE 4. PREFERRED REPORT ITEMS FOR SYSTEMATIC REVIEWS AND META-ANALYSIS FLOWCHART (PRISMA).

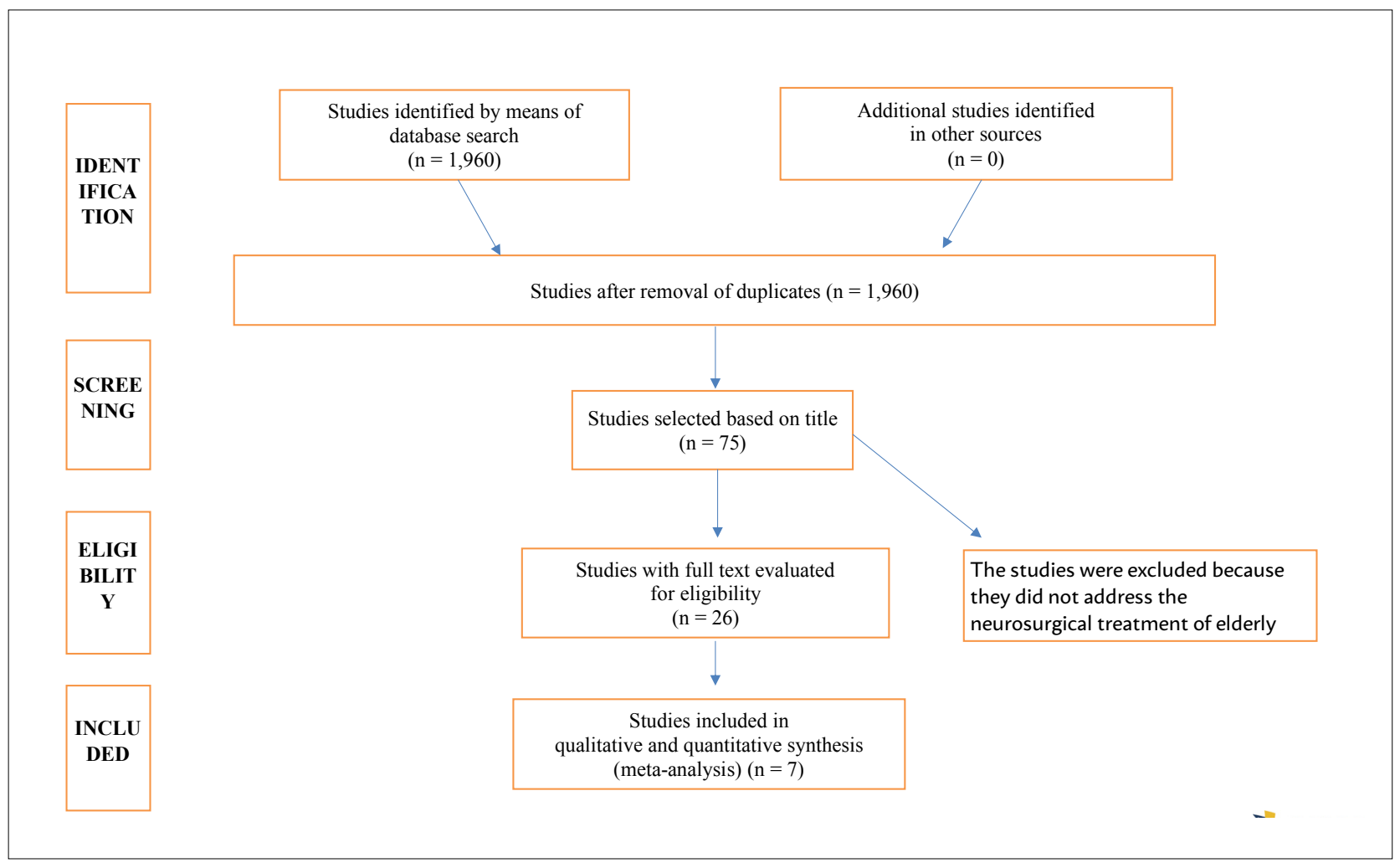


study was clearly established a priori. All studies included convenience samples, evaluated the entire sample treated as defined in the protocol and followed-up all patients until the outcome. All studies have had small losses of follow-up and followed-up the patients for sufficient time for the primary evaluated outcome (time of survival), which was the same in all studies. Therefore, the final analysis was not distorted. Since there was no variable for comparison, with the exception of different chemotherapy or radiotherapy regimens (which was not the objective of our study), all studies were considered non-comparative series of cases, with no variables for comparison. Considering the quality limit established by the Minors scale, which assigns 12 points to studies of good quality (non-comparative), we considered that the designs of all the studies had a good methodological quality.

\section{Application of evidence - \\ Recommendation}

The recommendations were designed by the re- view authors with the initial characteristic of the synthesis of evidence and were submitted to validation by all authors who participated in the creation of the guidelines.

\section{Conflict of interest}

There is no conflict of interest related to this review that can be declared by any of the authors.

\section{Final declaration}

The Guidelines Project, an initiative of the Brazilian Medical Association in partnership with the Specialty Societies, aims to reconcile medical information in order to standardize approaches that can aid the physician's reasoning and decision-making process. The information contained in this project must be submitted to the evaluation and criticism of the physician, responsible for the conduct to be followed, given the reality and clinical condition of each patient.

\section{REFERENCES}

1. Stupp R, Hegi ME, Mason WP, van den Bent MJ, Taphoorn MJ, Janzer $R C$, et al: Effects of radiotherapy with concomitant and adjuvant temozolomide versus radiotherapy alone on survival in glioblastoma in a randomized phase III study: 5-year analysis of the EORTC-NCIC trial. Lancet Oncol 10: 459-66, 2009

2. Ewelt C, Goeppert M, Rapp M, Steiger HJ, Stummer W, Sabel M: Glioblastoma multiforme of the elderly: the prognostic effect of resection on survival. J Neurooncol 103: 611-8, 2011. PMID: 20953662

3. Gessler F, Bernstock JD, Braczynski A, Lescher S, Baumgarten P, Harter PN, et al: Surgery for Glioblastoma in Light of Molecular Markers: Impact of Resection and MGMT Promoter Methylation in Newly Diagnosed IDH-1 Wild-Type Glioblastomas. Neurosurgery Published Online First: 2018/04/05. doi: 10.1093/neuros/nyy049: 2018

4. Haj A, Doenitz C, Schebesch KM, Ehrensberger D, Hau P, Putnik K, et al: Extent of Resection in Newly Diagnosed Glioblastoma: Impact of a Specialized Neuro-Oncology Care Center. Brain Sci 8: 2017

5. Stockhammer F: Treatment of glioblastoma in elderly patients. CNS Oncol 3: 159-67, 2014

6. Hoffermann M, Bruckmann L, Kariem Mahdy A, Asslaber M, Payer F, von Campe G: Treatment results and outcome in elderly patients with glioblastoma multiforme--a retrospective single institution analysis. Clin Neurol Neurosurg 128: 60-9, 2015. PMID: 25462098

7. Oszvald A, Guresir E, Setzer M, Vatter H, Senft C, Seifert V, et al: Glioblastoma therapy in the elderly and the importance of the extent of resection regardless of age. J Neurosurg 116: 357-64, 2012. PMID: 21942727

8. Lombardi G, Pace A, Pasqualetti F, Rizzato S, Faedi M, Anghileri E, et al: Predictors of survival and effect of short ( $40 \mathrm{~Gy}$ ) or standard-course (60

Gy) irradiation plus concomitant temozolomide in elderly patients with glioblastoma: a multicenter retrospective study of AINO (Italian Association of Neuro-Oncology). I Neurooncol 125: 359-67, 2015. PMID: 26423801

9. Gerstein J, Franz K, Steinbach JP, Seifert V, Fraunholz I, Weiss C, Rödel C.Postoperative radiotherapy and concomitant temozolomide for elderly patients with glioblastoma. Radiother Oncol. 2010 Dec:97(3):382-6. doi:10.1016/j.radonc.2010.06.014. Epub 2010 Sep 17. PubMed PMID: 20850883. PMID: 20850883

10. Pretanvil JA, Salinas IQ, Piccioni DE: Glioblastoma in the elderly: treatment patterns and survival. CNS Oncol 6: 19-28, 2017. PMID: 28001088

11. Bohman LE1, Gallardo I, Hankinson TC, Waziri AE, Mandigo CE, McKhann GM 2nd, Sisti MB, Canoll P, Bruce JN. The survival impact of postoperative infection in patients with glioblastoma multiforme.Neurosurgery. 2009 May;64(5):828-34; discussion 834-5. doi: 10.1227/01. NEU.0000343525.89321.C5. PMID: 19404146

12. Harris G, Jayamanne D, Wheeler H, Gzell C, Kastelan M, Schembri G, et al: Survival Outcomes of Elderly Patients With Glioblastoma Multiforme in Their 75th Year or Older Treated With Adjuvant Therapy. Int J Radiat Oncol Biol Phys 98: 802-10, 2017. PMID: 28602411

13. Wick A, Kessler T, Elia AEH, Winkler F, Batchelor TT, Platten M, et al: Glioblastoma in elderly patients: solid conclusions built on shifting sand? Neuro Oncol 20: 174-83, 2018

14. Guyatt GH, Oxman AD, Kunz R, Atkins D, Brozek J, Vist G, et al: GRADE guidelines: 2 . Framing the question and deciding on important outcomes. J Clin Epidemiol 64: 395-400, 2011 